\title{
ON REPRESENTATIONS OF ERROR TERMS RELATED TO THE DERIVATIVES FOR SOME DIRICHLET SERIES
}

\author{
JUN FURUYA, T. MAKOTO MINAMIDE AND YOSHIO TANIGAWA
}

\begin{abstract}
In previous papers, we examined several properties of an error term in a certain divisor problem related to the derivatives of the Riemann zeta-function. In this paper, we obtain representations of error terms related to the derivatives of some Dirichlet series, which can be regarded as generalized versions of a Dirichlet divisor problem and a Gauss circle problem. We also give the upper bounds of the error terms in terms of exponent pairs.
\end{abstract}

\section{Introduction and statement of results}

Let $\zeta(s)$ denote the Riemann zeta-function, and $\zeta^{(k)}(s)$ denote the $k$ th derivative of $\zeta(s)$ with $\zeta^{(0)}(s)=\zeta(s)$. Further let $D_{(k, l)}(n)$ be the coefficient of Dirichlet series $(-1)^{k+l} \zeta^{(k)}(s) \zeta^{(l)}(s)$ in $\Re s>1$ for any non-negative integers $k$ and $l$, namely

$$
D_{(k, l)}(n)=\sum_{d \mid n}(\log d)^{k}\left(\log \frac{n}{d}\right)^{l} .
$$

In the previous works, we investigated the upper bound estimates for the error term $\Delta_{(k, l)}(x)$, which is the error term on the summatory function $\sum_{n \leq x} D_{(k, l)}(n)$. In particular, we treated the upper bound estimates for the case $k=l$ in [15], and general $k$ and $l$ in [7]. As other properties for this error term, the truncated Voronoï-type formula and a mean square formula for $\Delta_{(1,1)}(x)$ were derived in [15]. Furthermore, the Riesz means and differences between two kinds of mean values of the error term were treated in [2].

In [7], we derived the representation of $\Delta_{(k, l)}(x)$ called the "Chowla-Walum formula", that is, the sum of the periodic Bernoulli function. Actually, we

Received April 3, 2017; received in final form October 16, 2017.

This work is supported by JSPS KAKENHI Grant Numbers 26400030, 15K17512 and $15 \mathrm{~K} 04778$.

2010 Mathematics Subject Classification. 11N37. 
proved that

$$
\Delta_{(k, l)}(x)=-\left\{R_{k, l}(x)+R_{l, k}(x)\right\}+O\left((\log x)^{k+l}\right)
$$

with

$$
R_{\alpha, \beta}(x)=\sum_{j=0}^{\beta}\left(\begin{array}{l}
\beta \\
j
\end{array}\right)(-1)^{j}(\log x)^{\beta-j} \sum_{n \leq \sqrt{x}} \psi\left(\frac{x}{n}\right)(\log n)^{\alpha+j}
$$

where $\left(\begin{array}{c}k \\ j\end{array}\right)$ is the binomial coefficient and $\psi(x)=x-[x]-1 / 2$ is the periodic Bernoulli function. Here $[x]$ denotes the greatest integer not exceeding $x$.

The formula (1.1) is derived by using the "Dirichlet hyperbola method", which is formulated as

$$
\begin{aligned}
\sum_{m n \leq x} f(n) g(m)= & \sum_{n \leq x^{c}} f(n) \sum_{m \leq x / n} g(m)+\sum_{n \leq x^{1-c}} g(n) \sum_{m \leq x / n} f(m) \\
& -\left(\sum_{n \leq x^{c}} f(n)\right)\left(\sum_{n \leq x^{1-c}} g(n)\right)
\end{aligned}
$$

for any arithmetical functions $f$ and $g$, where $c$ is a real number with $0 \leq c \leq 1$ (see [1, Theorem 3.17]).

For an arithmetical function $f(n)$, we put $L(s)=\sum_{n=1}^{\infty} f(n) n^{-s}$ for $\Re s>$ $\sigma_{f}$, where $\sigma_{f}$ is an abscissa of absolute convergence of this series. Then we have

$$
\zeta^{(k)}(s) L(s)=\sum_{n=1}^{\infty} \frac{d_{(k)}(n ; f)}{n^{s}}
$$

for $\Re s>\max \left(1, \sigma_{f}\right)$, where

$$
d_{(k)}(n ; f)=\sum_{d \mid n} f(d)\left(\log \frac{n}{d}\right)^{k}
$$

The aim of this paper is to study the summatory function

$$
\sum_{n \leq x} d_{(k)}(n ; f)
$$

and derive the Chowla-Walum type formula for the error term of (1.5). In particular, we treat two examples; one is $L(s)=(-1)^{l} \zeta^{(l)}(s-a)(-1<a<0)$, and the other is $L(s)=(-1)^{l} L^{(l)}(s, \chi)$ ( $\chi$ is the Dirichlet character mod 4$)$.

1.1. The case $L(s)=(-1)^{l} \zeta^{(l)}(s-a)$. For a real number $a(-1<a<0)$, let $\sigma_{a}(n)$ denote the arithmetical function defined by

$$
\sigma_{a}(n)=\sum_{d \mid n} d^{a}
$$


This is the coefficient of the Dirichlet series $\zeta(s) \zeta(s-a)$ for $\Re s>1$. A generalized divisor problem is to study the behaviour of the error term $\Delta_{a}(x)$ defined by

$$
\Delta_{a}(x)=\sum_{n \leq x} \sigma_{a}(n)-\zeta(1-a) x-\frac{\zeta(1+a)}{1+a} x^{1+a} .
$$

The Chowla-Walum type formula of $\Delta_{a}(x)$ is of the form

$$
\Delta_{a}(x)=-\sum_{n \leq \sqrt{x}} n^{a} \psi\left(\frac{x}{n}\right)-x^{a} \sum_{n \leq \sqrt{x}} n^{-a} \psi\left(\frac{x}{n}\right)+O(1)
$$

(cf. e.g. [6]). There are many researches on $\Delta_{a}(x)$, for example, the upper bound estimates, Voronoï-type representations and mean value formulas for $\Delta_{a}(x)$. We note that the studies of the function $\Delta_{a}(x)$ for $-1<a<0$ are deeply connected with the behaviour of the Riemann zeta function $\zeta(s)$ for $1 / 2<\sigma<1$. For the details of these topics, see [3], [4], [5], [6], [16].

Now, we shall consider a divisor problem for $(-1)^{k+l} \zeta^{(k)}(s) \zeta^{(l)}(s-a)$ with $-1<a<0$ (the case of $a=0$ was already studied in [7]). In this case, $f(n)=$ $n^{a}(\log n)^{l}$ in (1.4), and we put

$$
\sigma_{(k, l, a)}(n):=d_{(k)}(n ; f)=\sum_{d \mid n} d^{a}(\log d)^{l}\left(\log \frac{n}{d}\right)^{k} .
$$

We consider the error term of $\sum_{n \leq x} \sigma_{(k, l, a)}(n)$ and obtain the following theorem.

THEOREM 1.1. Let $\sigma_{(k, l, a)}(n)$ be the arithmetical function defined by (1.7). Then we have

$$
\sum_{n \leq x} \sigma_{(k, l, a)}(n)=x P_{k}(\log x)+x^{1+a} Q_{l}(\log x)+\Delta_{(k, l, a)}(x),
$$

where $P_{k}(x)$ and $Q_{l}(x)$ are certain polynomials in $x$ of degree $k$ and $l$ respectively, whose coefficients depend on $k, l$ and $a$, and $\Delta_{(k, l, a)}(x)$ is the error term defined by

$$
\Delta_{(k, l, a)}(x)=R_{(k, l)}(x ; a)+x^{a} R_{(l, k)}(x ;-a)+O\left((\log x)^{k+l}\right)
$$

with

$$
R_{(p, q)}(x ; r)=-\sum_{j=0}^{p}\left(\begin{array}{l}
p \\
j
\end{array}\right)(-1)^{j}(\log x)^{p-j} \sum_{n \leq \sqrt{x}} n^{r} \psi\left(\frac{x}{n}\right)(\log n)^{q+j} .
$$

We can see that the formula in Theorem 1.1 implies the previous formula (1.6).

As a direct application of Theorem 1.1, using the theory of exponent pairs, we obtain the following corollary, which is the non-trivial estimate of $\Delta_{(k, l, a)}(x)$. For the theory of exponent pairs, refer to [10], [13] and [14]. 
Corollary 1.2. Let $(\kappa, \lambda)$ be any exponent pair. Then we have

$$
\Delta_{(k, l, a)}(x) \ll \begin{cases}x^{\frac{a}{2}+\frac{\lambda+\kappa}{2(\kappa+1)}}(\log x)^{k+l+1} & \text { for } a+\frac{\lambda-\kappa}{\kappa+1}>0, \\ x^{\frac{(1+a) \kappa}{1-\lambda+2 \kappa}}(\log x)^{k+l+2} & \text { for } a+\frac{\lambda-\kappa}{\kappa+1} \leq 0 .\end{cases}
$$

Specific exponent pairs give several upper bound estimates of $\Delta_{(k, l, a)}(x)$ under some condition on $a$. For example, if we take $(\kappa, \lambda)=(11 / 82,57 / 82)$, we have

$$
\Delta_{(k, l,-1 / 2)}(x)=O\left(x^{11 / 94}(\log x)^{k+l+2}\right)
$$

$(11 / 94=0.11702 \ldots)$. We shall discuss this topic in Section 5 .

1.2. The case $L(s)=(-1)^{l} L^{(l)}(s, \chi)$. Let $\chi(n)$ be the primitive Dirichlet character modulo 4 , and $L(s, \chi)$ be the Dirichlet $L$-function associated to $\chi$ defined by

$$
L(s, \chi)=\sum_{n=1}^{\infty} \chi(n) n^{-s} \quad(\Re s>1) .
$$

The Gauss circle problem is to study the error term related to the Dirichlet series $4 \zeta(s) L(s, \chi)$. Indeed, let $P(x)$ denote the error term in the circle problem defined by

$$
P(x)=\sum_{1 \leq n \leq x} r(n)-\pi x,
$$

where $r(n)$ denotes the number of integer solutions of Diophantine equation $x^{2}+y^{2}=n$. The main object of the Gauss circle problem is to find the best possible estimation of $P(x)$. There are many results concerning several estimations of $P(x)$ and related topics on the circle problem. See e.g. [14] and [13] in details.

The Chowla-Walum type formula for $P(x)$ is of the form

$$
\begin{aligned}
P(x)= & -4 \sum_{n \leq \sqrt{x}} \chi(n) \psi\left(\frac{x}{n}\right)-4 \sum_{n \leq \sqrt{x}} \psi\left(\frac{x-n}{4 n}\right) \\
& +4 \sum_{n \leq \sqrt{x}} \psi\left(\frac{x-3 n}{4 n}\right)+O(1)
\end{aligned}
$$

(cf. [10, Theorem 4.8]).

Now we treat the arithmetical function $r_{(k, l)}(n)$ defined by

$$
r_{(k, l)}(n)=\sum_{d \mid n} \chi(d)(\log d)^{l}\left(\log \frac{n}{d}\right)^{k},
$$

which is the coefficient of the Dirichlet series $(-1)^{k+l} \zeta^{(k)}(s) L^{(l)}(s, \chi)$. In [8], we studied several arithmetical properties of the error term of the sum $\sum_{n \leq x} r_{(1,1)}(n)$. Actually we derived the truncated Voronoï formula, the mean square formula and the non-trivial estimate of the error term. 
Here, we shall derive the Chowla-Walum type formula of the error term related to the summatory function $\sum_{n \leq x} r_{(k, l)}(n)$ for general $k$ and $l$. We obtain the following theorem.

THEOREM 1.3. Let $r_{(k, l)}(n)$ be the arithmetical function defined by (1.8). Then we have

$$
\sum_{n \leq x} r_{(k, l)}(n)=-x Q_{k}(\log x ; k, l)+P_{(k, l)}(x)+O\left((\log x)^{k+l}\right),
$$

where $Q_{d}(x ; k, l)$ is the polynomial in $x$ of degree $d$ whose coefficients depend on $k$ and $l,{ }^{1}$ and $P_{(k, l)}(x)$ is the error term defined by

$$
\begin{aligned}
P_{(k, l)}(x)= & -\sum_{j=0}^{k}\left(\begin{array}{c}
k \\
j
\end{array}\right)(-1)^{j}(\log x)^{k-j} \sum_{n \leq \sqrt{x}} \chi(n) \psi\left(\frac{x}{n}\right)(\log n)^{l+j} \\
& +R_{(k, l)}^{(1)}(x ; \chi)+R_{(k, l)}^{(3)}(x ; \chi)+O\left((\log x)^{k+l}\right)
\end{aligned}
$$

with

$$
R_{(k, l)}^{(\alpha)}(x ; \chi)=\chi(-\alpha) \sum_{j=0}^{l}\left(\begin{array}{l}
l \\
j
\end{array}\right)(-1)^{j}(\log x)^{l-j} \sum_{n \leq \sqrt{x}} \psi\left(\frac{y-\alpha n}{4 n}\right)(\log n)^{k+j}
$$

for $\alpha=1$ and 3 .

As an application of Theorem 1.3, we obtain the following corollary.

Corollary 1.4. Let $(\kappa, \lambda)$ be any exponent pair. Under the notations of Theorem 1.3, we have

$$
P_{(k, l)}(x) \ll \begin{cases}x^{1 / 3}(\log x)^{k+l} & \text { if } \kappa=\lambda=1 / 2, \\ x^{\frac{\kappa+\lambda}{2(\kappa+1)}}(\log x)^{k+l}+|P(x)|(\log x)^{k+l} & \text { if } \kappa \neq \lambda .\end{cases}
$$

In particular, the exponent pair $(\kappa, \lambda)=(97 / 251,132 / 251)$ gives the estimate

$$
P_{(k, l)}(x)=O\left(x^{\frac{229}{696}}(\log x)^{k+l}\right) .
$$

Note that $229 / 696=0.329022 \ldots$ In the case $k=l=0$, the best estimate (Huxley [12]) at present is

$$
P_{(0,0)}(x)=O\left(x^{\frac{131}{416}}(\log x)^{\frac{18627}{8320}}\right)
$$

$(131 / 416=0.314903 \ldots)$. In this case, our Corollary 1.4 is weaker than the above estimate.

1 Note that the leading term of $-x Q_{k}(\log x ; k, l)$ is positive, since $c_{f}(0)<0$ (cf. e.g. [9]). 


\section{Preliminaries}

As a preparation for the purpose, we consider the summation formulas involving the log-function. We present these formulas as the following lemmas.

Lemma 2.1 ([7, Lemma 1]). For a non-negative integer $q$, we have

$$
\begin{aligned}
\sum_{n \leq y}(\log n)^{q}= & y \sum_{j=0}^{q} a_{q}(j)(\log y)^{j}-\psi(y)(\log y)^{q} \\
& +q \int_{1}^{y} \frac{\psi(t)(\log t)^{q-1}}{t} d t+c_{q}
\end{aligned}
$$

where $a_{q}(j)=(-1)^{q+j} q ! / j$ ! and $c_{q}$ is given by

$$
c_{q}= \begin{cases}-1 / 2 & \text { if } q=0, \\ (-1)^{q+1} q ! & \text { if } q \geq 1 .\end{cases}
$$

Lemma 2.2. For a non-negative integer $q$ and a real number a with $-1<$ $a<0$, we have

$$
\begin{aligned}
\sum_{n \leq y} n^{a}(\log n)^{q}= & y^{1+a} \sum_{j=0}^{q} \frac{a_{q}(j)}{(1+a)^{q-j+1}}(\log y)^{j}-\psi(y) y^{a}(\log y)^{q} \\
& +\int_{1}^{y} t^{a-1}(a \log t+q) \psi(t)(\log t)^{q-1} d t+\frac{\tilde{c}_{q}}{(1+a)^{q+1}}
\end{aligned}
$$

with

$$
\tilde{c}_{q}= \begin{cases}(1-a) c_{0} & \text { if } q=0, \\ c_{q} & \text { if } q \geq 1,\end{cases}
$$

where $a_{q}(j)$ and $c_{q}$ are the constants defined in Lemma 2.1.

Proof. This lemma can be proved by the Euler-Maclaurin summation formula and an integral formula

$$
\int_{1}^{y} t^{a}(\log t)^{q} d t=y^{1+a} \sum_{j=0}^{q} \frac{a_{q}(j)}{(1+a)^{q-j+1}}(\log y)^{j}+\frac{(-1)^{q+1} q !}{(1+a)^{q+1}} .
$$

Note that the formula (2.1) is valid for the cases $a=0$ and $q=0$.

Now we shall transform $\sum_{n \leq x} d_{(k)}(n ; f)$ by the hyperbola method (1.2). By the definition of $d_{(k)}(n ; f)$ and (1.2) with $c=1 / 2$, we can see that

$$
\begin{aligned}
\sum_{n \leq x} d_{(k)}(n ; f)= & \sum_{n \leq \sqrt{x}} f(n) \sum_{m \leq x / n}(\log m)^{k} \\
& +\sum_{n \leq \sqrt{x}}(\log n)^{k} \sum_{m \leq x / n} f(m)
\end{aligned}
$$




$$
\begin{aligned}
& -\left(\sum_{n \leq \sqrt{x}} f(n)\right)\left(\sum_{n \leq \sqrt{x}}(\log n)^{k}\right) \\
= & S_{1}+\sum_{n \leq \sqrt{x}}(\log n)^{k} \sum_{m \leq x / n} f(m)-S_{2},
\end{aligned}
$$

say.

In $S_{1}$, we have by Lemma 2.1 that

$$
\begin{aligned}
S_{1}= & \sum_{n \leq \sqrt{x}} f(n)\left\{\frac{x}{n} \sum_{j=0}^{k} a_{k}(j)\left(\log \frac{x}{n}\right)^{j}-\psi\left(\frac{x}{n}\right)\left(\log \frac{x}{n}\right)^{k}\right. \\
& \left.+k \int_{1}^{x / n} \frac{\psi(t)(\log t)^{k-1}}{t} d t+c_{k}\right\} \\
=: & S_{11}+S_{12}+S_{13}+c_{k} \sum_{n \leq \sqrt{x}} f(n) .
\end{aligned}
$$

It is easy to see from the binomial expansion and partial summation that

$$
\begin{aligned}
S_{11}= & x \sum_{j=0}^{k} a_{k}(j) \sum_{\nu=0}^{j}\left(\begin{array}{l}
j \\
\nu
\end{array}\right)(-1)^{\nu}(\log x)^{j-\nu} \sum_{n \leq \sqrt{x}} \frac{1}{n} f(n)(\log n)^{\nu} \\
= & \left(\sqrt{x} \sum_{n \leq \sqrt{x}} f(n)\right) \sum_{j=0}^{k} \frac{a_{k}(j)}{2^{j}}(\log x)^{j} \\
& -x \sum_{j=0}^{k} a_{k}(j) \sum_{\nu=0}^{j}\left(\begin{array}{l}
j \\
\nu
\end{array}\right)(-1)^{\nu}(\log x)^{j-\nu} \int_{1}^{\sqrt{x}}\left\{\frac{1}{t}(\log t)^{\nu}\right\}^{\prime} \sum_{n \leq t} f(n) d t,
\end{aligned}
$$

where we have used the identity

$$
\sum_{n=0}^{N}\left(\begin{array}{c}
N \\
n
\end{array}\right) \frac{(-1)^{n}}{2^{n}}=\frac{1}{2^{N}}
$$

Similarly, we have

$$
S_{12}=-\sum_{j=0}^{k}\left(\begin{array}{l}
k \\
j
\end{array}\right)(-1)^{j}(\log x)^{k-j} \sum_{n \leq \sqrt{x}} f(n) \psi\left(\frac{x}{n}\right)(\log n)^{j} .
$$

In $S_{13}$, interchanging summation and integration we have

$$
\begin{aligned}
S_{13}= & k\left(\sum_{n \leq \sqrt{x}} f(n)\right) \int_{1}^{\sqrt{x}} \frac{\psi(t)(\log t)^{k-1}}{t} d t \\
& +k \int_{\sqrt{x}}^{x} \frac{\psi(t)(\log t)^{k-1}}{t} \sum_{n \leq x / t} f(n) d t .
\end{aligned}
$$


Furthermore, in $S_{2}$, we again apply the formula in Lemma 2.1 to obtain

$$
\begin{aligned}
S_{2}= & \left(\sqrt{x} \sum_{n \leq \sqrt{x}} f(n)\right) \sum_{j=0}^{k} \frac{a_{k}(j)}{2^{j}}(\log x)^{j} \\
& -2^{-k} \psi(\sqrt{x})(\log x)^{k} \sum_{n \leq \sqrt{x}} f(n) \\
& +k\left(\sum_{n \leq \sqrt{x}} f(n)\right) \int_{1}^{\sqrt{x}} \frac{\psi(t)(\log t)^{k-1}}{t} d t+c_{k} \sum_{n \leq \sqrt{x}} f(n) .
\end{aligned}
$$

Therefore, we obtain the following lemma.

LEMma 2.3. We have

$$
\begin{aligned}
\sum_{n \leq x} d_{(k)}(n ; f) & \\
= & -x \sum_{j=0}^{k} a_{k}(j) \sum_{\nu=0}^{j}\left(\begin{array}{l}
j \\
\nu
\end{array}\right)(-1)^{\nu}(\log x)^{j-\nu} \\
& \times \int_{1}^{\sqrt{x}}\left\{\frac{1}{t}(\log t)^{\nu}\right\} \sum_{n \leq t} f(n) d t \\
& -\sum_{j=0}^{k}\left(\begin{array}{c}
k \\
j
\end{array}\right)(-1)^{j}(\log x)^{k-j} \sum_{n \leq \sqrt{x}} f(n) \psi\left(\frac{x}{n}\right)(\log n)^{j} \\
& +k \int_{\sqrt{x}}^{x} \frac{\psi(t)(\log t)^{k-1}}{t} \sum_{n \leq x / t} f(n) d t+\sum_{n \leq \sqrt{x}}(\log n)^{k} \sum_{m \leq x / n} f(m) \\
& +2^{-k} \psi(\sqrt{x})(\log x)^{k} \sum_{n \leq \sqrt{x}} f(n) .
\end{aligned}
$$

From now on, we assume some conditions of $\sum_{n \leq x} f(n)$. Actually, we put

$$
\sum_{n \leq x} f(n)=g(x)+E(x),
$$

where $g(x)$ is the "main term" and $E(x)$ is the "error term". We assume that the function $g(x)$ is continuously differentiable and $E(x)=O\left(x^{\theta_{1}}(\log x)^{\theta_{2}}\right)$, where $\theta_{1}$ is a constant with $-1<\theta_{1} \leq 0$ and $\theta_{2}$ is a non-negative integer. Further we assume that the mean value of $E(x)$ is of the form

$$
\int_{1}^{x} E(t) d t=A_{f} x+B_{f}+O\left(x^{\theta_{3}}(\log x)^{\theta_{4}}\right)
$$

with some constants $A_{f}, B_{f}, \theta_{3}$ and a non-negative integer $\theta_{4}$. Note that $A_{f}=0$ if $\theta_{1}<0$, and $B_{f}$ is included in the $O$-term if $\theta_{3} \geq 0$. 
Now we shall transform the formula (2.3) under the assumption (2.4). Indeed, we have

$$
\begin{aligned}
\sum_{n \leq x} d_{(k)}(n ; f) & \\
= & -x \sum_{j=0}^{k} a_{k}(j) \sum_{\nu=0}^{j}\left(\begin{array}{l}
j \\
\nu
\end{array}\right)(-1)^{\nu}(\log x)^{j-\nu} \int_{1}^{\sqrt{x}}\left\{\frac{1}{t}(\log t)^{\nu}\right\}^{\prime} g(t) d t \\
& -\sum_{j=0}^{k}\left(\begin{array}{l}
k \\
j
\end{array}\right)(-1)^{j}(\log x)^{k-j} \sum_{n \leq \sqrt{x}} f(n) \psi\left(\frac{x}{n}\right)(\log n)^{j} \\
& +k \int_{\sqrt{x}}^{x} \frac{\psi(t)(\log t)^{k-1}}{t} g\left(\frac{x}{t}\right) d t+\sum_{n \leq \sqrt{x}}(\log n)^{k} g\left(\frac{x}{n}\right) \\
& +2^{-k} \psi(\sqrt{x})(\log x)^{k} g(\sqrt{x})+T(x)
\end{aligned}
$$

with

$$
\begin{aligned}
T(x)= & -x \sum_{j=0}^{k} a_{k}(j) \sum_{\nu=0}^{j}\left(\begin{array}{l}
j \\
\nu
\end{array}\right)(-1)^{\nu}(\log x)^{j-\nu} \\
& \times \int_{1}^{\sqrt{x}}\left\{\frac{1}{t}(\log t)^{\nu}\right\}^{\prime} E(t) d t \\
& +k \int_{\sqrt{x}}^{x} \frac{\psi(t)(\log t)^{k-1}}{t} E\left(\frac{x}{t}\right) d t+\sum_{n \leq \sqrt{x}}(\log n)^{k} E\left(\frac{x}{n}\right) \\
& +2^{-k} \psi(\sqrt{x})(\log x)^{k} E(\sqrt{x}) \\
= & T_{1}+T_{2}+\sum_{n \leq \sqrt{x}}(\log n)^{k} E\left(\frac{x}{n}\right)+T_{3},
\end{aligned}
$$

say. By the assumption $E(x)=O\left(x^{\theta_{1}}(\log x)^{\theta_{2}}\right)$ with the constants $\theta_{j}$ defined in $(2.4)$, it is trivially seen that

$$
\begin{aligned}
T_{2} & \ll x^{\theta_{1}}(\log x)^{\theta_{2}+k-1} \int_{\sqrt{x}}^{x} t^{-1-\theta_{1}} d t \\
& \ll(\log x)^{\theta_{2}+k-1} \times \begin{cases}\log x & \text { if } \theta_{1}=0, \\
x^{\theta_{1} / 2} & \text { if }-1<\theta_{1}<0\end{cases}
\end{aligned}
$$

and $T_{3}=O\left(x^{\theta_{1} / 2}(\log x)^{\theta_{2}+k}\right)$. Hence, we have $T_{2} \ll T_{3}$.

On $T_{1}$, we see that $\int_{\sqrt{x}}^{\infty}\left\{\frac{1}{t}(\log t)^{\nu}\right\}^{\prime} E(t) d t=O\left(x^{-\left(1-\theta_{1}\right) / 2}(\log x)^{\theta_{2}+\nu}\right)$ by the assumption of $E(x)$, and therefore $\int_{1}^{\infty}\left\{\frac{1}{t}(\log t)^{\nu}\right\}^{\prime} E(t) d t$ is convergent. More precisely, by the assumption $\int_{1}^{x} E(t) d t=A_{f} x+B_{f}+O\left(x^{\theta_{3}}(\log x)^{\theta_{4}}\right)$ 
and integration by parts, we can see that

$$
\begin{aligned}
& \int_{\sqrt{x}}^{\infty}\left\{\frac{1}{t}(\log t)^{\nu}\right\}^{\prime} E(t) d t \\
& \quad=-\left.\left\{\frac{1}{t}(\log t)^{\nu}\right\}^{\prime}\right|_{t=\sqrt{x}} \int_{1}^{\sqrt{x}} E(t) d t-\int_{\sqrt{x}}^{\infty}\left\{\frac{1}{t}(\log t)^{\nu}\right\}^{\prime \prime} \int_{1}^{t} E(u) d u d t \\
& \quad=-A_{f} x^{-1 / 2}(\log \sqrt{x})^{\nu}+O\left(x^{-1+\theta_{3} / 2}(\log x)^{\theta_{4}+\nu}\right) .
\end{aligned}
$$

Hence by putting $c_{f}(\nu)=\int_{1}^{\infty}\left\{\frac{1}{t}(\log t)^{\nu}\right\}^{\prime} E(t) d t$ we have

$$
\begin{aligned}
T_{1}= & -x \sum_{j=0}^{k} a_{k}(j) \sum_{\nu=0}^{j}\left(\begin{array}{l}
j \\
\nu
\end{array}\right)(-1)^{\nu}(\log x)^{j-\nu} c_{f}(\nu) \\
& -A_{f} x^{1 / 2} \sum_{j=0}^{k} \frac{a_{k}(j)}{2^{j}}(\log x)^{j}+O\left(x^{\theta_{3} / 2}(\log x)^{\theta_{4}+k}\right)
\end{aligned}
$$

by $(2.2)$.

Combining all the above results, we obtain

$$
\begin{aligned}
T(x)= & -x \sum_{j=0}^{k} a_{k}(j) \sum_{\nu=0}^{j}\left(\begin{array}{c}
j \\
\nu
\end{array}\right)(-1)^{\nu}(\log x)^{j-\nu} c_{f}(\nu) \\
& -A_{f} x^{1 / 2} \sum_{j=0}^{k} \frac{a_{k}(j)}{2^{j}}(\log x)^{j}+\sum_{n \leq \sqrt{x}}(\log n)^{k} E\left(\frac{x}{n}\right) \\
& +O\left(x^{\theta_{1} / 2}(\log x)^{\theta_{2}+k}\right)+O\left(x^{\theta_{3} / 2}(\log x)^{\theta_{4}+k}\right) .
\end{aligned}
$$

As for the second preliminary, we transform the fourth term on the righthand side in (2.5). We put this part as $U$. By partial summation, we have

$$
\begin{aligned}
U & =g(\sqrt{x}) \sum_{n \leq \sqrt{x}}(\log n)^{k}+x \int_{1}^{\sqrt{x}} t^{-2} g^{\prime}\left(\frac{x}{t}\right) \sum_{n \leq t}(\log n)^{k} d t \\
& =U_{1}+U_{2},
\end{aligned}
$$

say. As for the function $U_{1}$, we have by Lemma 2.1 that

$$
\begin{aligned}
U_{1}= & g(\sqrt{x})\left\{\sqrt{x} \sum_{j=0}^{k} \frac{a_{k}(j)}{2^{j}}(\log x)^{j}-\psi(\sqrt{x})(\log \sqrt{x})^{k}\right. \\
& \left.+k \int_{1}^{\sqrt{x}} \frac{\psi(t)(\log t)^{k-1}}{t} d t+c_{k}\right\} \\
= & U_{11}-g(\sqrt{x}) \psi(\sqrt{x})(\log \sqrt{x})^{k}+U_{12}+c_{k} g(\sqrt{x}) .
\end{aligned}
$$

Note that the second term on the right-hand side in the above is canceled by the fifth term on the right-hand side in (2.5). 
On $U_{2}$, we have

$$
\begin{aligned}
U_{2}= & \int_{\sqrt{x}}^{x} g^{\prime}(t)\left\{\frac{x}{t} \sum_{j=0}^{k} a_{k}(j)\left(\log \frac{x}{t}\right)^{j}-\psi\left(\frac{x}{t}\right)\left(\log \frac{x}{t}\right)^{k}\right. \\
& \left.+k \int_{1}^{x / t} \frac{\psi(u)(\log u)^{k-1}}{u} d u+c_{k}\right\} d t \\
= & U_{21}-\int_{\sqrt{x}}^{x} g^{\prime}(t) \psi\left(\frac{x}{t}\right)\left(\log \frac{x}{t}\right)^{k} d t \\
& +U_{22}+c_{k}\{g(x)-g(\sqrt{x})\}
\end{aligned}
$$

say. It is easy to see that

$$
U_{22}=k \int_{1}^{\sqrt{x}} \frac{\psi(u)(\log u)^{k-1}}{u} g\left(\frac{x}{u}\right) d u-U_{12} .
$$

On $U_{21}$, we have

$$
\begin{aligned}
U_{21}= & x \sum_{j=0}^{k} a_{k}(j) \sum_{\nu=0}^{j}\left(\begin{array}{l}
j \\
\nu
\end{array}\right)(-1)^{\nu}(\log x)^{j-\nu} \int_{\sqrt{x}}^{x} g^{\prime}(t) \frac{(\log t)^{\nu}}{t} d t \\
= & -U_{11}-x \sum_{j=0}^{k} a_{k}(j) \sum_{\nu=0}^{j}\left(\begin{array}{l}
j \\
\nu
\end{array}\right)(-1)^{\nu}(\log x)^{j-\nu} \\
& \times \int_{\sqrt{x}}^{x} g(t)\left\{\frac{1}{t}(\log t)^{\nu}\right\}^{\prime} d t+a_{k}(0) g(x)
\end{aligned}
$$

by (2.2) and the formula

$$
\sum_{n=0}^{N}\left(\begin{array}{l}
N \\
n
\end{array}\right)(-1)^{n}= \begin{cases}1 & \text { if } N=0 \\
0 & \text { if } N \geq 1\end{cases}
$$

Collecting these estimates we have

$$
\begin{aligned}
U= & -x \sum_{j=0}^{k} a_{k}(j) \sum_{\nu=0}^{j}\left(\begin{array}{l}
j \\
\nu
\end{array}\right)(-1)^{\nu}(\log x)^{j-\nu} \int_{\sqrt{x}}^{x} g(t)\left\{\frac{1}{t}(\log t)^{\nu}\right\}^{\prime} d t \\
& +k \int_{1}^{\sqrt{x}} \frac{\psi(u)(\log u)^{k-1}}{u} g\left(\frac{x}{u}\right) d u-\int_{\sqrt{x}}^{x} g^{\prime}(t) \psi\left(\frac{x}{t}\right)\left(\log \frac{x}{t}\right)^{k} d t \\
& -2^{-k} g(\sqrt{x}) \psi(\sqrt{x})(\log x)^{k}+\left(a_{k}(0)+c_{k}\right) g(x) .
\end{aligned}
$$

Therefore, as the preparation of the proofs, we obtain the following lemma. 
LEMMA 2.4. Let $d_{(k)}(n ; f)$ be the function defined by (1.4). Under the assumption (2.4), we have

$$
\begin{aligned}
\sum_{n \leq x} d_{(k)}(n ; f) & \\
= & -x \sum_{j=0}^{k} a_{k}(j) \sum_{\nu=0}^{j}\left(\begin{array}{l}
j \\
\nu
\end{array}\right)(-1)^{\nu}(\log x)^{j-\nu} \int_{1}^{x}\left\{\frac{1}{t}(\log t)^{\nu}\right\}^{\prime} g(t) d t \\
& +k \int_{1}^{x} \frac{\psi(t)(\log t)^{k-1}}{t} g\left(\frac{x}{t}\right) d t \\
& -\int_{\sqrt{x}}^{x} g^{\prime}(t) \psi\left(\frac{x}{t}\right)\left(\log \frac{x}{t}\right)^{k} d t \\
& -\sum_{j=0}^{k}\left(\begin{array}{l}
k \\
j
\end{array}\right)(-1)^{j}(\log x)^{k-j} \sum_{n \leq \sqrt{x}} f(n) \psi\left(\frac{x}{n}\right)(\log n)^{j} \\
& +\left(a_{k}(0)+c_{k}\right) g(x)+T(x)
\end{aligned}
$$

with

$$
\begin{aligned}
T(x)= & -x \sum_{j=0}^{k} a_{k}(j) \sum_{\nu=0}^{j}\left(\begin{array}{l}
j \\
\nu
\end{array}\right)(-1)^{\nu}(\log x)^{j-\nu} c_{f}(\nu) \\
& -A_{f} x^{1 / 2} \sum_{j=0}^{k} \frac{a_{k}(j)}{2^{j}}(\log x)^{j}+\sum_{n \leq \sqrt{x}}(\log n)^{k} E\left(\frac{x}{n}\right) \\
& +O\left(x^{\theta_{1} / 2}(\log x)^{\theta_{2}+k}\right)+O\left(x^{\theta_{3} / 2}(\log x)^{\theta_{4}+k}\right) .
\end{aligned}
$$

\section{Proof of Theorem 1.1}

Let $f(n)=n^{a}(\log n)^{l}$ and $\sigma_{(k, l, a)}(n)=d_{(k)}(n ; f)$. By Lemma 2.2 we find that the main term $g(y)$ and the error term $E(y)$ in $\sum_{n \leq y} f(n)$ are of the forms

$$
g(y)=y^{1+a} \sum_{j=0}^{l} \frac{a_{l}(j)}{(1+a)^{l-j+1}}(\log y)^{j}
$$

and

$$
\begin{aligned}
E(y)= & -y^{a} \psi(y)(\log y)^{l}+\int_{1}^{y} t^{a-1}(a \log t+l) \psi(t)(\log t)^{l-1} d t \\
& +\frac{\tilde{c}_{l}}{(1+a)^{l+1}},
\end{aligned}
$$

respectively. From the definition of $a_{l}(j)$, we find that

$$
g^{\prime}(y)=y^{a}(\log y)^{l} .
$$


Since the integral on the right-hand side of $E(y)$ converges when $y \rightarrow \infty$ we have the following expressions:

$$
\begin{aligned}
E(y) & =A_{f}-y^{a} \psi(y)(\log y)^{l}-\int_{y}^{\infty} t^{a-1}(a \log t+l) \psi(t)(\log t)^{l-1} d t \\
& =A_{f}+O\left(y^{a}(\log y)^{l}\right),
\end{aligned}
$$

and

$$
\int_{1}^{y} E(t) d t=A_{f} y+B_{f}+O\left(y^{a}(\log y)^{l}\right)
$$

where

$$
A_{f}=\frac{\tilde{c}_{l}}{(1+a)^{l+1}}+\int_{1}^{\infty} t^{a-1}(a \log t+l) \psi(t)(\log t)^{l-1} d t
$$

and

$$
B_{f}=-A_{f}-\int_{1}^{\infty} t^{a} \psi(t)(\log t)^{l} d t-\int_{1}^{\infty} t^{a}(a \log t+l) \psi(t)(\log t)^{l-1} d t .
$$

Hence, we can take $\theta_{1}=\theta_{2}=0, \theta_{3}=a$ and $\theta_{4}=l$. We remark that $\theta_{3}=a<0$ in this case (compare with the choice in Section 6).

Now we shall consider the formula (2.8).

The fourth term on the right-hand side in (2.8) coincides with $R_{(k, l)}(x ; a)$.

By (3.1), (3.2) and integration by parts we find that

$$
\begin{aligned}
\int_{1}^{x} & \left\{\frac{1}{t}(\log t)^{\nu}\right\}^{\prime} g(t) d t \\
= & {\left[\frac{(\log t)^{\nu}}{t} g(t)\right]_{1}^{x}-\int_{1}^{x} t^{a-1}(\log t)^{\nu+l} d t } \\
= & (\log x)^{\nu} x^{-1} g(x)-\delta_{\nu}-\int_{1}^{\infty} t^{a-1}(\log t)^{\nu+l} d t \\
& +\int_{x}^{\infty} t^{a-1}(\log t)^{\nu+l} d t,
\end{aligned}
$$

where $\delta_{\nu}=0$ if $\nu \geq 1$ and $\delta_{0}=g(1)$. We should note that the integral in the second and third lines are convergent by the condition $a<0$. Hence, the first term on the right-hand side in (2.8) becomes

$$
\begin{aligned}
= & -g(x) \sum_{j=0}^{k} a_{k}(j)(\log x)^{j} \sum_{\nu=0}^{j}\left(\begin{array}{l}
j \\
\nu
\end{array}\right)(-1)^{\nu} \\
& +x \sum_{j=0}^{k} a_{k}(j) \sum_{\nu=0}^{j}\left(\begin{array}{l}
j \\
\nu
\end{array}\right)(-1)^{\nu}(\log x)^{j-\nu}\left(\delta_{\nu}+\int_{1}^{\infty} t^{a-1}(\log t)^{\nu+l} d t\right) \\
& -x \sum_{j=0}^{k} a_{k}(j) \sum_{\nu=0}^{j}\left(\begin{array}{l}
j \\
\nu
\end{array}\right)(-1)^{\nu}(\log x)^{j-\nu} \int_{x}^{\infty} t^{a-1}(\log t)^{\nu+l} d t .
\end{aligned}
$$


The first line on the right-hand side of (3.5) equals to $-g(x) a_{k}(0)$ since the sum over $\nu$ vanishes for $j \geq 1$. We consider the third line of (3.5), which we denote by $J(x)$. By putting back the binomial expansion we have

$$
J(x)=-x \sum_{j=0}^{k} a_{k}(j) \int_{x}^{\infty} t^{a-1}\left(\log \frac{x}{t}\right)^{j}(\log t)^{l} d t .
$$

If we change the variable by $\frac{x}{t}=u$, we have

$$
\begin{aligned}
J(x)= & -x^{1+a} \sum_{j=0}^{k} a_{k}(j) \int_{0}^{1} u^{-a-1}(\log u)^{j}\left(\log \frac{x}{u}\right)^{l} d u \\
= & -x^{1+a} \sum_{j=0}^{k} a_{k}(j) \sum_{\nu=0}^{l}\left(\begin{array}{l}
l \\
\nu
\end{array}\right)(-1)^{\nu}(\log x)^{l-\nu} \\
& \times \int_{0}^{1} u^{-a-1}(\log u)^{j+\nu} d u .
\end{aligned}
$$

Hence by (3.5) and (3.6), we can see that the first term on the right-hand side of (2.8) has the form

$$
x \sum_{j=0}^{k} A_{1, j}(a, k, l)(\log x)^{j}+x^{1+a} \sum_{j=0}^{l} A_{2, j}(a, k, l)(\log x)^{j} .
$$

Next, we treat the second term on the right-hand side in (2.8). Let $\psi_{1}(y)=$ $\int_{1}^{y} \psi(t) d t$. Noting that $\psi_{1}(y)=O(1)$ uniformly in $y$, we have by integration by parts that

$$
\begin{aligned}
k \int_{1}^{x} \frac{\psi(t)(\log t)^{k-1}}{t} g\left(\frac{x}{t}\right) d t= & -k \int_{1}^{x} \psi_{1}(t)\left\{\frac{(\log t)^{k-1}}{t} g\left(\frac{x}{t}\right)\right\}^{\prime} d t \\
& +O\left(x^{-1}(\log x)^{k-1}\right) .
\end{aligned}
$$

By (3.1), we get

$$
\begin{aligned}
& \frac{(\log t)^{k-1}}{t} g\left(\frac{x}{t}\right) \\
& =x^{1+a} \sum_{j=0}^{l} \frac{a_{l}(j)}{(1+a)^{l-j+1}} \sum_{\nu=0}^{j}\left(\begin{array}{l}
j \\
\nu
\end{array}\right)(-1)^{\nu}(\log x)^{j-\nu} \frac{(\log t)^{\nu+k-1}}{t^{2+a}},
\end{aligned}
$$

and thus

$$
\begin{aligned}
& k \int_{1}^{x} \frac{\psi(t)(\log t)^{k-1}}{t} g\left(\frac{x}{t}\right) d t \\
& \quad=-k x^{1+a} \sum_{j=0}^{l} \frac{a_{l}(j)}{(1+a)^{l-j+1}} \sum_{\nu=0}^{j}\left(\begin{array}{l}
j \\
\nu
\end{array}\right)(-1)^{\nu}(\log x)^{j-\nu}
\end{aligned}
$$




$$
\begin{aligned}
& \times \int_{1}^{x} \psi_{1}(t)\left\{\frac{(\log t)^{\nu+k-1}}{t^{2+a}}\right\}^{\prime} d t \\
& +O\left(x^{-1}(\log x)^{k-1}\right) .
\end{aligned}
$$

Since $-1<a<0$, we see that the integral in the above is convergent absolutely. We obtain

$$
\begin{aligned}
k \int_{1}^{x} \frac{\psi(t)(\log t)^{k-1}}{t} g\left(\frac{x}{t}\right) d t= & x^{1+a} \sum_{j=0}^{l} A_{3, j}(a, k, l)(\log x)^{j} \\
& +O\left(x^{-1}(\log x)^{k+l-1}\right)
\end{aligned}
$$

As for the third one, we have by noting the formula (3.2) that

$$
\begin{aligned}
\int_{\sqrt{x}}^{x} g^{\prime}(t) \psi\left(\frac{x}{t}\right)\left(\log \frac{x}{t}\right)^{k} d t \\
=x \int_{1}^{\sqrt{x}} u^{-2} \psi(u) g^{\prime}\left(\frac{x}{u}\right)(\log u)^{k} d u \\
=x^{1+a} \int_{1}^{\sqrt{x}} u^{-2-a} \psi(u)\left(\log \frac{x}{u}\right)^{l}(\log u)^{k} d u \\
=x^{1+a} \sum_{\nu=0}^{l}\left(\begin{array}{l}
l \\
\nu
\end{array}\right)(-1)^{\nu}(\log x)^{l-\nu} \\
\quad \times \int_{1}^{\sqrt{x}} u^{-2-a} \psi(u)(\log u)^{\nu+k} d u .
\end{aligned}
$$

Since

$$
\begin{aligned}
\int_{1}^{\sqrt{x}} u^{-2-a} \psi(u)(\log u)^{\nu+k} d u= & \int_{1}^{\infty} u^{-2-a} \psi(u)(\log u)^{\nu+k} d u \\
& +O\left(x^{-1-a / 2}(\log x)^{\nu+k}\right)
\end{aligned}
$$

we get

$$
\begin{aligned}
& \int_{\sqrt{x}}^{x} g^{\prime}(t) \psi\left(\frac{x}{t}\right)\left(\log \frac{x}{t}\right)^{k} d t \\
& =x^{1+a} \sum_{j=0}^{l} A_{4, j}(a, k, l)(\log x)^{j}+O\left(x^{a / 2}(\log x)^{k+l}\right) .
\end{aligned}
$$

Clearly $a_{k}(0) g(x)$ in the fifth term of (2.8) cancels with the first line of (3.5), hence it becomes $c_{k} g(x)$, which we write

$$
c_{k} g(x)=x^{1+a} \sum_{j=0}^{l} A_{5, j}(a, k, l)(\log x)^{j} .
$$


It remains to consider the formula of (2.9). By the definition of $E(x)$, we have

$$
\begin{aligned}
\sum_{n \leq \sqrt{x}}(\log n)^{k} E\left(\frac{x}{n}\right)= & \sum_{n \leq \sqrt{x}}(\log n)^{k}\left\{-\left(\frac{x}{n}\right)^{a} \psi\left(\frac{x}{n}\right)\left(\log \frac{x}{n}\right)^{l}\right. \\
& \left.+\int_{x / n}^{\infty} t^{a-1}(a \log t+l) \psi(t)(\log t)^{l-1} d t+A_{f}\right\} \\
= & V_{1}+V_{2}+V_{3},
\end{aligned}
$$

say. For $V_{1}$, it is easy to see that

$$
V_{1}=-x^{a} \sum_{\nu=0}^{l}\left(\begin{array}{l}
l \\
\nu
\end{array}\right)(-1)^{\nu}(\log x)^{l-\nu} \sum_{n \leq \sqrt{x}} n^{-a} \psi\left(\frac{x}{n}\right)(\log n)^{k+\nu},
$$

which coincides with $x^{a} R_{(l, k)}(x ;-a)$. Also it is easily seen from Lemma 2.1 that

$$
V_{3}=A_{f} \sqrt{x} \sum_{j=0}^{k} a_{k}(j)(\log \sqrt{x})^{k}+O\left((\log x)^{k}\right) .
$$

For $V_{2}$, changing the summation and integration, we have

$$
\begin{aligned}
V_{2}= & \int_{\sqrt{x}}^{x} t^{a-1}(a \log t+l) \psi(t)(\log t)^{l-1} \sum_{x / t<n \leq \sqrt{x}}(\log n)^{k} d t \\
& +\left(\sum_{n \leq \sqrt{x}}(\log n)^{k}\right) \int_{x}^{\infty} t^{a-1}(a \log t+l) \psi(t)(\log t)^{l-1} d t \\
= & -\int_{\sqrt{x}}^{x} t^{a-1}(a \log t+l) \psi(t)(\log t)^{l-1} \sum_{n \leq x / t}(\log n)^{k} d t \\
& +\left(\sum_{n \leq \sqrt{x}}(\log n)^{k}\right) \int_{\sqrt{x}}^{\infty} t^{a-1}(a \log t+l) \psi(t)(\log t)^{l-1} d t \\
= & V_{21}+V_{22},
\end{aligned}
$$

say. It is easy to see that $V_{22} \ll x^{a / 2}(\log x)^{k+l}$. For $V_{21}$, we apply Lemma 2.2 and get

$$
\begin{aligned}
V_{21}= & -x \sum_{j=0}^{k} a_{k}(j) \int_{\sqrt{x}}^{x} t^{a-2}(a \log t+l) \psi(t)(\log t)^{l-1}\left(\log \frac{x}{t}\right)^{j} d t \\
& +O\left(x^{a / 2}(\log x)^{k+l}\right) \\
= & -x \sum_{j=0}^{k} a_{k}(j) \sum_{\nu=0}^{j}\left(\begin{array}{l}
j \\
\nu
\end{array}\right)(-1)^{\nu}(\log x)^{j-\nu}
\end{aligned}
$$




$$
\begin{aligned}
& \times \int_{\sqrt{x}}^{x} t^{a-2}(a \log t+l) \psi(t)(\log t)^{l-1+\nu} d t \\
&+O\left(x^{a / 2}(\log x)^{k+l}\right) \\
& \ll x^{a / 2}(\log x)^{k+l} .
\end{aligned}
$$

Collecting these estimates we obtain that

$$
\begin{aligned}
\sum_{n \leq \sqrt{x}}(\log n)^{k} E\left(\frac{x}{n}\right)= & x^{a} R_{(l, k)}(x ;-a)+A_{f} \sqrt{x} \sum_{j=0}^{k} a_{k}(j)(\log \sqrt{x})^{k} \\
& +O\left((\log x)^{k}\right)+O\left(x^{a / 2}(\log x)^{k+l}\right) .
\end{aligned}
$$

The second term on the right-hand side above is canceled by the second term on the right-hand side in (2.9). Clearly the first term of $T(x)$ has the form $x \sum_{j=0}^{k} A_{6, j}(a, k, l)(\log x)^{j}$.

Collecting all formulas, we obtain the assertion of Theorem 1.1.

REMARK. We can see that the coefficient of $x(\log x)^{k}$, which is the greatest term for the main term of $\sum_{n \leq x} \sigma_{(k, l, a)}(n)$, does not vanish. Actually, we can see this fact as follows: The explicit value of such coefficient is equal to the sum of the terms from $A_{1, k}(a, k, l)$ and $A_{6, k}(a, k, l)$ in the case $j=k$ and $\nu=0$. By the definitions of $A_{1, k}(a, k, l)$ and $A_{6, k}(1, k, l)$, we have that the corresponding term coming from $A_{1, k}(a, k, l)$ is

$$
=\frac{(-1)^{l} l !}{(1+a)^{l+1}}+\int_{1}^{\infty} t^{a-1}(\log t)^{l} d t
$$

and the term corresponding to $A_{6, k}(a, k, l)$ is

$$
=-c_{f}(0)=(-1)^{l-1} \zeta^{(l)}(1-a)-\frac{(-1)^{l} l !}{(1+a)^{l}}-\int_{1}^{\infty} t^{a-1}(\log t)^{l} d t .
$$

Then we have that the coefficient of $x(\log x)^{k}$ is $(-1)^{l-1} \zeta^{(l)}(1-a)$. We can trivially see that $\zeta^{(l)}(1-a) \neq 0$ for all non-negative integer $l$ and the real number $a$ with $-1<a<0$, hence we see that the coefficient of $x(\log x)^{k}$ is not equal to zero.

\section{Proof of Corollary $\mathbf{1 . 2}$}

In order to prove Corollary 1.2, we apply the formula of $\psi(x)$ in the following lemma.

LEMMA 4.1 ([11, p. 245]). Let $h$ be any real number. We have

$$
\psi(x)=-\frac{1}{2 \pi i} \sum_{1 \leq|h| \leq H} \frac{e(h x)}{h}+O\left(E_{H}(x)\right)
$$


with $e(y)=\exp (2 \pi i y)$, where $E_{H}(x)$ is the function estimated and represented as

$$
E_{H}(x)=\min \left(1, \frac{1}{H\|x\|}\right)=\sum_{h=-\infty}^{\infty} b(h) e(h x)
$$

with

$$
b(0) \ll \frac{\log H}{H} \quad \text { and } \quad b(h) \ll \min \left(\frac{\log H}{H}, \frac{H}{h^{2}}\right) \quad(h \neq 0) .
$$

By using this lemma, we have the following lemma.

LEMmA 4.2. Let $r$ be a real number and let $\mathfrak{g}_{N}(x)$ be the function defined by

$$
\mathfrak{g}_{N}(x)=\sum_{N<n \leq 2 N} n^{r} \psi\left(\frac{x}{n}\right)
$$

with $1 \leq N \leq \sqrt{x}$. Then we have

$$
\mathfrak{g}_{N}(x) \ll x^{\frac{\kappa}{\kappa+1}} N^{r+\frac{\lambda-\kappa}{\kappa+1}} \log x
$$

for any exponent pair $(\kappa, \lambda)$.

Proof. Substituting (4.1) in (4.3), we have

$$
\mathfrak{g}_{N}(x)=-\frac{1}{2 \pi i} \sum_{1 \leq|h| \leq H} \frac{1}{h} \sum_{N<n \leq 2 N} e\left(\frac{h x}{n}\right)+O\left(\sum_{N<n \leq 2 N} n^{r} E_{H}\left(\frac{x}{n}\right)\right) .
$$

Let $(\kappa, \lambda)$ be an exponent pair. By partial summation and applying this exponent pair, we get

$$
\begin{aligned}
\sum_{N<n \leq 2 N} n^{r} e\left(\frac{h x}{n}\right) & \ll \max _{N<t \leq 2 N}\left|\sum_{N<n \leq t} e\left(\frac{h x}{n}\right)\right| N^{r} \\
& \ll N^{r}\left\{\left(\frac{h x}{N^{2}}\right)^{\kappa}(t-N)^{\lambda}+\frac{N^{2}}{h x}\right\} \\
& \ll h^{\kappa} x^{\kappa} N^{a+\lambda-2 \kappa} .
\end{aligned}
$$

Hence the first term on the right-hand side of (4.5) is evaluated as as

$$
\ll H^{\kappa} x^{\kappa} N^{a+\lambda-2 \kappa} \times \begin{cases}\log H & \kappa=0, \\ 1 & \kappa>0 .\end{cases}
$$

On the other hand by using (4.2), the second term on the right-hand side of (4.5) is bounded as

$$
\sum_{N<n \leq 2 N} n^{r} E_{H}\left(\frac{x}{n}\right) \ll\left(x^{\kappa} N^{a+\lambda-2 \kappa} H^{\kappa}+\frac{N^{a+1}}{H}\right) \log H .
$$


Hence, we get

$$
\mathfrak{g}_{N}(x) \ll\left(x^{\kappa} N^{a+\lambda-2 \kappa} H^{\kappa}+\frac{N^{a+1}}{H}\right) \log H
$$

for $H \geq 1$. Note that $\mathfrak{g}_{N}(x) \ll N^{a+1}$ trivially, hence (4.6) holds for all $H>0$. Taking $H=x^{-\kappa /(\kappa+1)} N^{(1-\lambda+2 \kappa) /(\kappa+1)}$, we get (4.4).

Proof of Corollary 1.2. Let $r$ be a real number and let

$$
G(x, r)=\sum_{n \leq \sqrt{x}} n^{r} \psi\left(\frac{x}{n}\right)(\log x)^{A}
$$

for a non-negative integer $A$. In view of Theorem 1.1, we have to estimate the upper bounds of $G(x, a)$ and $x^{a} G(x,-a)$.

Let

$$
g(t)=\sum_{n \leq t} n^{r} \psi\left(\frac{x}{n}\right) .
$$

By partial summation, we have

$$
\begin{aligned}
G(x, r) & =g(\sqrt{x})(\log \sqrt{x})^{A}-A \int_{1}^{\sqrt{x}} g(t)(\log t)^{A-1} \frac{1}{t} d t \\
& \ll\left\{\max _{t \leq \sqrt{x}}|g(t)|\right\}(\log x)^{A} .
\end{aligned}
$$

Hence, it is enough to evaluate $|g(t)|$ for $t$ in the range $1 \leq t \leq \sqrt{x}$. Let $N_{j}=t / 2^{j}$. Then by the standard decomposition technique we have

$$
g(t)=\sum_{j=1}^{j_{0}} \mathfrak{g}_{N_{j}}(x)+O(1)
$$

where $j_{0}=[\log t / \log 2]$.

For $G(x, a)$ we take $r=a$ for $-1<a<0$ in (4.4). We consider the three cases.

Case 1. Suppose that $a+\frac{\lambda-\kappa}{\kappa+1}>0$. In this case, we have

$$
\begin{aligned}
g(t) & \ll x^{\frac{\kappa}{\kappa+1}} \sum_{j}\left(\frac{t}{2^{j}}\right)^{a+\frac{\lambda-\kappa}{\kappa+1}} \log x \\
& \ll x^{\frac{\kappa}{\kappa+1}} t^{a+\frac{\lambda-\kappa}{\kappa+1}} \log x .
\end{aligned}
$$

Case 2. Suppose that $a+\frac{\lambda-\kappa}{\kappa+1}<0$. Let $t$ be a real number such that $0<t<1$. By the theory of exponent pairs,

$$
\left(\kappa_{t}, \lambda_{t}\right)=t(0,1)+(1-t)(\kappa, \lambda)=((1-t) \kappa, t / 2+(1-t) \lambda)
$$


is also an exponent pair. If we take

$$
t_{0}=\frac{-a(\kappa+1)-(\lambda-\kappa)}{1-a \kappa-(\lambda-\kappa)},
$$

then we have $a\left(\kappa_{t_{0}}+1\right)+\lambda_{t_{0}}-\kappa_{t_{0}}=0$. We also note that $0<t_{0}<1$ by the assumption $a+\frac{\lambda-\kappa}{\kappa+1}<0$. Applying this exponent pair in (4.4), we get

$$
\mathfrak{g}_{N}(x) \ll x^{\frac{\kappa_{0}}{\kappa_{0}+1}} \log x=x^{\frac{(1+a) \kappa}{1-\lambda+2 \kappa}} \log x,
$$

and hence

$$
g(t) \ll x^{\frac{(1+a) \kappa}{1-\lambda+2 \kappa}}(\log x)^{2} .
$$

Case 3. Suppose that $a+\frac{\lambda-\kappa}{\kappa+1}=0$. As in the case 2, we get

$$
g(t) \ll x^{\frac{\kappa}{\kappa+1}}(\log x)^{2} .
$$

From these estimates, we have

$$
G(x, a) \ll \begin{cases}x^{\frac{a}{2}+\frac{\lambda+\kappa}{2(\kappa+1)}}(\log x)^{A+1}, & a+\frac{\lambda-\kappa}{\kappa+1}>0, \\ x^{\frac{(1+a) \kappa}{1-\lambda+2 \kappa}}(\log x)^{A+2}, & a+\frac{\lambda-\kappa}{\kappa+1} \leq 0 .\end{cases}
$$

For $x^{a} G(x,-a)$ we take $r=-a>0$ in (4.4). Since $-a+\frac{\lambda-\kappa}{\kappa+1}>1$ we have

$$
x^{a} G(x,-a) \ll x^{\frac{a}{2}+\frac{\lambda+\kappa}{2(\kappa+1)}}(\log x)^{A+1} .
$$

Therefore, by the definition of $\Delta_{(k, l, a)}(x)$ in Theorem 1.1, we obtain

$$
\Delta_{(k, l, a)}(x) \ll \begin{cases}x^{\frac{a}{2}+\frac{\lambda+\kappa}{2(\kappa+1)}}(\log x)^{k+l+1}, & a+\frac{\lambda-\kappa}{\kappa+1}>0, \\ x^{\frac{(1+a) \kappa}{1-\lambda+2 \kappa}}(\log x)^{k+l+2}+x^{\frac{a}{2}+\frac{\lambda+\kappa}{2(\kappa+1)}}(\log x)^{k+l+1}, & a+\frac{\lambda-\kappa}{\kappa+1} \leq 0 .\end{cases}
$$

Remarking that $\frac{a}{2}+\frac{\lambda+\kappa}{2(\kappa+1)} \leq \frac{(1+a) \kappa}{1-\lambda+2 \kappa}$ for $a+\frac{\lambda-\kappa}{\kappa+1} \leq 0$, we obtain the assertion of Corollary 1.2.

\section{Some remarks on Corollary $\mathbf{1 . 2}$}

We first recall that the constant $a$ satisfies the condition $-1<a<0$.

If we take the trivial exponent pair $(\kappa, \lambda)=(0,1)$, we have $a+\frac{\lambda-\kappa}{\kappa+1}>0$ and hence

$$
\Delta_{(k, l, a)}(x)=O\left(x^{\frac{a+1}{2}}(\log x)^{k+l+1}\right)
$$

for $-1<a<0$. Similarly, if we take $(\kappa, \lambda)=(1 / 2,1 / 2)$, we have $a+\frac{\lambda-\kappa}{\kappa+1} \leq 0$ and hence

for $-1<a<0$.

$$
\Delta_{(k, l, a)}(x)=O\left(x^{\frac{a+1}{3}}(\log x)^{k+l+1}\right)
$$

Here are some other examples. Each exponent pair is taken from [14].

(i) Put $(\kappa, \lambda)=(1 / 6,4 / 6)$, then

$$
\Delta_{(k, l, a)}(x) \ll \begin{cases}x^{\frac{a}{2}+\frac{5}{14}}(\log x)^{k+l+1} & \text { for }-\frac{3}{7}<a<0 \\ x^{\frac{(a+1)}{4}}(\log x)^{k+l+2} & \text { for }-1<a \leq-\frac{3}{7}\end{cases}
$$


(ii) Put $(\kappa, \lambda)=(2 / 18,13 / 18)$, then

$$
\Delta_{(k, l, a)}(x) \ll \begin{cases}x^{\frac{a}{2}+\frac{3}{8}}(\log x)^{k+l+1} & \text { for }-\frac{11}{20}<a<0, \\ x^{\frac{2}{9}(a+1)}(\log x)^{k+l+2} & \text { for }-1<a \leq-\frac{11}{20} .\end{cases}
$$

(iii) Put $(\kappa, \lambda)=(11 / 82,57 / 82)$, then

$$
\Delta_{(k, l, a)}(x) \ll \begin{cases}x^{\frac{a}{2}+\frac{34}{93}}(\log x)^{k+l+1} & \text { for }-\frac{46}{93}<a<0, \\ x^{\frac{11(a+1)}{47}}(\log x)^{k+l+2} & \text { for }-1<a \leq-\frac{46}{93} .\end{cases}
$$

Thus, the third one gives the special estimate

$$
\Delta_{(k, l,-1 / 2)}(x)=O\left(x^{\frac{11}{94}}(\log x)^{k+l+2}\right) .
$$

\section{Proof of Theorem $\mathbf{1 . 3}$}

We put $f(n)=\chi(n)(\log n)^{l}$, then $d_{(k)}(n ; f)=r_{(k, l)}(n)$ in this setting. By the formula

$$
\sum_{n \leq y} \chi(n)=\frac{1}{2}-\psi\left(\frac{y-1}{4}\right)+\psi\left(\frac{y-3}{4}\right)
$$

(cf. [6, Lemma 4.7]), we have by partial summation that

$$
\sum_{n \leq y} f(n)=\left\{-\psi\left(\frac{y-1}{4}\right)+\psi\left(\frac{y-3}{4}\right)\right\}(\log y)^{l}+A_{f}+O\left(y^{-1}(\log y)^{l-1}\right)
$$

where $A_{f}$ is a constant. Hence, we have

$$
\int_{1}^{y} \sum_{n \leq t} f(n) d t=A_{f} y+O\left((\log y)^{l}\right) .
$$

Thus if we put $g(x)=0, E(x)=\sum_{n \leq x} f(n), \theta_{1}=\theta_{3}=0$ and $\theta_{2}=\theta_{4}=l$, we can see that the function $f(n)$ of this setting satisfies all assumptions of the error term $E(x)$ in Section 2.

On this setting, the formula (2.5) is reduced to

$$
\begin{aligned}
\sum_{n \leq x} r_{(k, l)}(n)= & -\sum_{j=0}^{k}\left(\begin{array}{l}
k \\
j
\end{array}\right)(-1)^{j}(\log x)^{k-j} \sum_{n \leq \sqrt{x}} \chi(n) \psi\left(\frac{x}{n}\right)(\log n)^{l+j} \\
& -x \sum_{j=0}^{k} a_{k}(j) \sum_{\nu=0}^{j}\left(\begin{array}{l}
j \\
\nu
\end{array}\right)(-1)^{\nu}(\log x)^{j-\nu} c_{f}(\nu) \\
& -A_{f} x^{1 / 2} \sum_{j=0}^{k} \frac{a_{k}(j)}{2^{j}}(\log x)^{j}+\sum_{n \leq \sqrt{x}}(\log n)^{k} E\left(\frac{x}{n}\right) \\
& +O\left((\log x)^{k+l}\right) .
\end{aligned}
$$


Here the second term on the right-hand side contributes the main term $-x Q_{k}(\log x ; k, l)$ of the theorem; the first one above is the first term of $P_{(k, l)}(x)$.

On the fourth term in the right-hand side of the above formula, applying partial summation and substituting the formula (6.1) into it, we have

$$
\begin{aligned}
\sum_{n \leq \sqrt{x}}(\log n)^{k} E\left(\frac{x}{n}\right) & \\
= & \sum_{n \leq \sqrt{x}}(\log n)^{k} \sum_{m \leq x / n} \chi(m)(\log m)^{l} \\
= & \sum_{n \leq \sqrt{x}}(\log n)^{k}\left\{\left(-\psi\left(\frac{x / n-1}{4}\right)+\psi\left(\frac{x / n-3}{4}\right)\right)\left(\log \frac{x}{n}\right)^{l}+A_{f}\right\} \\
& +O\left((\log x)^{k+l-1}\right) \\
= & \sum_{j=0}^{l}\left(\begin{array}{l}
l \\
j
\end{array}\right)(-1)^{j}(\log x)^{l-j} \sum_{n \leq \sqrt{x}}\left\{-\psi\left(\frac{x-n}{4 n}\right)+\psi\left(\frac{x-3 n}{4 n}\right)\right\}(\log n)^{k+j} \\
& +A_{f}\left(x^{1 / 2} \sum_{j=0}^{k} \frac{a_{k}(j)}{2^{j}}(\log x)^{j}+O\left((\log x)^{k}\right)\right)+O\left((\log x)^{k+l-1}\right) .
\end{aligned}
$$

Hence, the term containing $A_{f}$ cancels and the remaining terms containing $\psi(x)$ contribute $R_{(k, l)}^{(1)}(x, \chi)$ and $R_{(k, l)}^{(3)}(x, \chi)$ of $P_{(k, l)}(x)$ of the theorem. The proof of Theorem 1.3 is complete.

The assertions of Corollary 1.4 can be proved by using Theorem 1.3 and the method used in [10] and [7, Section 5]. We omit the details of the proof of this corollary.

Acknowledgments. The authors would like to thank the referees for their careful reading and useful comments to this paper.

\section{REFERENCES}

[1] T. M. Apostol, Introduction to analytic number theory, Undergraduate Texts in Mathematics, Springer-Verlag, New York-Heidelberg, 1976. MR 0434929

[2] D. Banerjee and M. Minamide, The average behaviour of the error term in a new kind of the divisor problem, J. Math. Anal. Appl. 438 (2016), no. 2, 533-550. MR 3466051

[3] X. Cao, J. Furuya, Y. Tanigawa and W. Zhai, A generalized divisor problem and the sum of Chowla and Walum, J. Math. Anal. Appl. 400 (2013), 15-21. MR 3003959

[4] X. Cao, J. Furuya, Y. Tanigawa and W. Zhai, A generalized divisor problem and the sum of Chowla and Walum II, Funct. Approx. Comment. Math. 49 (2013), 159-188. MR 3127905

[5] X. Cao, Y. Tanigawa and W. Zhai, On a conjecture of Chowla and Walum, Sci. China Math. 53 (2010), no. 10, 2755-2771. MR 2728277

[6] S. Chowla, Contributions to the analytic theory of numbers, Math. Z. 35 (1932), 279299. MR 1545300 
[7] J. Furuya, M. Minamide and Y. Tanigawa, Representations and evaluations of the error term in a certain divisor problem, Math. Slovaca 66 (2016), 575-582. MR 3543721

[8] J. Furuya, M. Minamide and Y. Tanigawa, On a new circle problem, J. Aust. Math. Soc. 103 (2017), 231-249. MR 3703924

[9] J. Furuya and Y. Tanigawa, On integrals and Dirichlet series obtained from the error term in the circle problem, Funct. Approx. Comment. Math. 51 (2014), no. 2, 303-333. MR 3282630

[10] S. W. Graham and G. Kolesnik, Van der Corput's method for exponential sums, London Mathematical Society Lecture Note Series, vol. 126, Cambridge University Press, Cambridge, 1991. MR 1145488

[11] D. R. Heath-Brown, The Piateckǐ̌-S̆apiro prime number theory, J. Number Theory 16 (1983), 242-266. MR 0698168

[12] M. N. Huxley, Exponential sums and lattice points III, Proc. Lond. Math. Soc. 87 (2003), 591-609. MR 2005876

[13] A. Ivić, The Riemann zeta-function: Theory and applications, Dover Publications, Inc., Mineola, NY, 2003, Reprint of the 1985 original (Wiley). MR 1994094

[14] E. Krätzel, Lattice points, Kluwer Acad. Publishers, Dordrecht, 1988. MR 0998378

[15] M. Minamide, The truncated Voronoï formula for the derivative of the Riemann zeta function, Indian J. Math. 55 (2013), no. 3, 325-352. MR 3137009

[16] Y.-F. S. Pétermann, Divisor problems and exponent pairs, Arch. Math. 50 (1988), 243-250. MR 0933919

Jun Furuya, Department of Integrated Human Sciences (Mathematics), Hamamatsu University School of Medicine, Hamamatsu, 431-3192, Japan

E-mail address: jfuruya@hama-med.ac.jp

T. Makoto Minamide, Graduate School of Sciences and Technology for InnoVation, Yamaguchi University, Yamaguchi, 753-8512, Japan

E-mail address: minamide@yamaguchi-u.ac.jp

Yoshio Tanigawa, Graduate School of Mathematics, Nagoya University, NAGOYA, 464-8602, JAPAN

E-mail address: tanigawa@math.nagoya-u.ac.jp 